Check for updates

Cite this: Mater. Adv., 2021, 2, 4652

Received 3rd March 2021 Accepted 1st June 2021

DOI: $10.1039 / \mathrm{d} 1 \mathrm{ma} 00188 \mathrm{~d}$

rsc.li/materials-advances

\title{
A slow and sustained release of methotrexate (MTX) from a new polymeric dicalcium phosphate dehydrate cement (P-DCPD)
}

\begin{abstract}
Rahul Vaidya, ${ }^{a}$ Emily J Ren, ${ }^{a}$ Tong Shi, ${ }^{b}$ Angelica Gardia (D) ${ }^{b}$ and Weiping Ren (D) *b
A novel and injectable polymeric dicalcium phosphate dehydrate cement (P-DCPD) was developed that is mechanically strong and has excellent cohesion. Methotrexate (MTX) is an anticancer drug for many tumors including bone cancer. The current study assessed the performance of MTX loaded P-DCPD (MTX-P) in vitro. The applied properties of MTX-P include MTX release, effects on cell viability of human breast cancer MCF 7 cells and murine osteoblastic MC3T3 cells. The impacts of MTX-P on the injectability, setting time, material microstructure and mechanical strength were also evaluated. A slow and sustained MTX release from MTX-P was observed. Eluents collected from MTX-P were cytotoxic to MCF7 cells. MT3T3 cells, however, were much less sensitive to MTX-containing eluents. MTX-P was injectable. A remarkable delay of the setting time was observed in $0.5 \%$ MTX-P ( $45.7 \pm 1.9 \mathrm{~min}$ ) and $1 \%$ MTX-P (49.5 $\pm 3.4 \mathrm{~min})$ as compared to control-P (11.8 $\pm 1.2 \mathrm{~min})$, respectively. The compressive strength was significantly reduced in $0.5 \% \mathrm{MTX}-\mathrm{P}(16.49 \pm 2.43 \mathrm{MPa})$ and in $1 \% \mathrm{MTX}-\mathrm{P}(10.68 \pm$ 3.97 $\mathrm{MPa})$ as compared to control-P $(53.09 \pm 8.64 \mathrm{MPa})$. The inferior performance of MTX-P observed might be due to the insufficient crystal formation and uneven distribution of MTX within P-DCPD matrices. Our data may shed some light on the future application of MTX-P in the treatment of bone defects after tumor excision with a plethora of applications by including other anticancer drugs because of its safety and degradability. Further material optimization and in vivo validation are required to achieve a clinically applicable product.
\end{abstract}

\section{Introduction}

Bone metastases can be found in up to $70 \%$ of the cancer patients. ${ }^{1,2}$ Increased long-term survival of patients with cancer also increases the risk of bone metastases and pathological fractures. ${ }^{3}$ The success of internal fixation of impending or pathologic fractures following metastasis has a favourable impact on the quality of life of the patient. ${ }^{4}$ Anticancer drugloaded bone fillers have been used to fill bone defects after removal surgery, ${ }^{4,5}$ enhance implant stability, prevent local cancer progression and reconstructive device failure. ${ }^{6-8}$

Poly(methyl methacrylate) (PMMA) bone cements are the most commonly used bone void fillers for the local delivery of anticancer drugs. ${ }^{8-10}$ While acceptable, PMMA possesses several major limitations, ${ }^{11,12}$ including limited and burst drug release, ${ }^{6,13}$ toxic monomer release, ${ }^{14}$ high exothermic reaction and not degradable. ${ }^{8-10}$ Calcium phosphate cement (CPC) has been widely used as bone void filler. ${ }^{15}$ The end products of commercial CPCs

\footnotetext{
${ }^{a}$ Department of Orthopedics, Detroit Medical Center, Detroit, MI 48201, USA

${ }^{b}$ Department of Biomedical Engineering, Wayne State University, Detroit, MI 48201, USA. E-mail:as7606@wayne.edu
}

can be divided into two types: hydroxyapatite (HA) and dicalcium phosphate dehydrate (DCPD). ${ }^{15}$ DCPD cements have been proposed as an alternative to PMMA cements for the local delivery of anticancer drugs ${ }^{6,16}$ because of its injectability and slow degradation. ${ }^{17}$ Using CPC as local delivery of anticancer drugs is not clinically preferred ${ }^{18}$ because of some critical weaknesses, including poor cohesion, ${ }^{15}$ mechanically weak ${ }^{19}$ and burst drug release. ${ }^{20}$ Therefore, surgeons are actively looking for new bone void fillers that are biocompatible, mechanical strong, excellent anti-washout, and capable of delivering anticancer drugs in a sustained and controllable manner.

Polyphosphates are polyelectrolytes that play key roles in biological and material science. ${ }^{21}$ Calcium polyphosphate (CPP) is a polymeric inorganic ceramic composed of linear polyphosphate chains. ${ }^{22}$ We recently found that CPP hydrogel can be formed in the presence of water at optimized conditions. ${ }^{23} \mathrm{With}$ the CPP gelation technology, we developed a new way to prepare injectable polymeric DCPD-forming cement (P-DCPD) by the reaction of CPP gel with tetracalcium phosphate (TTCP). ${ }^{24}$ The setting reaction of P-DCPD is initiated by the interaction of numerous ionic binding sites of disentangled polyphosphate chains of CPP gel with ions of calcium and orthophosphate 
released from TTCP dissolution. ${ }^{24}$ The setting mechanism of P-DCPD is completely different from that of classical CPCs as described by Brown et al. in 1980s. ${ }^{25}$ To the best of our knowledge, no ceramic cement using similar technologies has been reported before. P-DCPD represents a new bone void filler with significant advantages, including strong mechanically strength, ${ }^{26}$ excellent cohesion, ${ }^{24}$ as well as a mean of sustained drug delivery $^{27,28}$ because of its unique ionic binding and intrinsic entanglement of polyphosphate chains with embedded drugs. ${ }^{29}$ The handling properties of P-DCPD is similar to PMMA cement and other commercially available CPCs. P-DCPD is mainly composed of calcium and phosphor and contains no cross-linkers or other additives. P-DCPD is expected to meet the unmet clinical needs as a better anticancer drug-eluting bone void filler for the load-bearing bone defects caused by bone cancer (primary and/or metastatic). Like DCPD, P-DCPD is osteoconductive and has similar mineral composition to that of native bone tissue. ${ }^{17,24,30}$

Methotrexate (MTX), an inhibitor of DNA synthesis, is one of the most commonly used anticancer drugs for human cancers including bone cancer (primary or secondary). ${ }^{31}$ However, its clinical efficacy is limited by its short plasma half-life ${ }^{32}$ and the risk of developing drug resistance. ${ }^{33}$ PMMA cements are not desirable carrier for local MTX delivery because of unsatisfactory clinical outcome ${ }^{34}$ caused by limited and burst drug release, non-degradability and the heating reaction during setting. ${ }^{35}$ Few studies have been conducted for the delivery of MTX via CPC system. ${ }^{36}$

The aim of this study was to determine the release of MTX from P-DCPD, and the influence of MTX loading on the cell viability of MCF 7 cells and osteoblastic MC3T3 cells. The impacts of MTX loading on the injectability, setting time and mechanical strength were also evaluated. In addition, the mechanisms of MTX release from P-DCPD cement were discussed.

\section{Experimental}

\section{Materials}

All chemicals used were of analytical grade and purchased from Sigma-Aldrich (USA) if not stated otherwise. Human breast cancer MCF-7 (ATCC ${ }^{\circledR}$ HTB22 $^{\mathrm{TM}}$ ) cell line and murine MC3T3-E1 pre-osteoblast cell line were purchased from ATCC (Manassas, VA). Alpha-modified minimum essential medium $(\alpha-\mathrm{MEM})$ and Dulbecco's modified Eagle's medium (DMEM) was purchased from Invitrogen (Carlsbad, CA). Methotrexate (MTX) and MTX assay ELISA kit were from Enzo Life sciences (Farmingdale, NY 11735).

\section{Preparation of P-DCPD and MTX loading}

CPP gel was prepared as described elsewhere. ${ }^{23}$ The TTCP powder was prepared by solid-state reaction of dicalcium phosphate anhydrous (DCPA, $\mathrm{CaHPO}_{4}$ ) and calcium carbonate (CaCO3) (Sigma-Aldrich) by heating at $1500{ }^{\circ} \mathrm{C}$ for $18 \mathrm{~h}$. P-DCPD was prepared by mixing CPP gel with TTCP $(1: 0.87, \mathrm{w} / \mathrm{w})$ at room temperature for setting. ${ }^{24}$ P-DCPD was doped with MTX at a ratio of $0 \%, 0.5 \%$ and $1 \%$, respectively (wt/wt), by adding MTX powders into CPP gel prior to mixing with TCPP for setting.

\section{Setting time and injectability}

The setting time was recorded using standard Gillmore needle method. ${ }^{24}$ The injectability was measured using an ASTM F45108 standard intrusion test mold. ${ }^{24}$

\section{MicroCT analysis}

The porosity, pore size and pore size distribution of MTX-P scaffolds were analyzed by MicroCT (Scanco Viva CT 40, Scanco Medical, Switzerland) with a voltage of $70 \mathrm{kVp}$ and a current of $114 \mu \mathrm{A}$ at $10 \mu \mathrm{m}$ resolution. A cylindrical volume of interest was selected (54 slices) for each scaffold, and an optimal threshold of 300 was determined. The microstructure of the scaffolds was determined using built-in software to measure the pore wall thickness (Tb.Th), pore size (Tb.Sp), porosity, pore size and distribution.

\section{Scanning electron microscopy}

The morphology of MTX-P scaffold surface was characterized by scanning electron microscopy (SEM) (Quanta FEG450, FEI, Hillsboro, OR, USA). Scaffolds were coated with gold (Gold Sputter, Effa Coater, USA) before SEM analysis. Morphologies were viewed at $10 \mathrm{kV}$ accelerating voltage.

\section{Mechanical testing}

Cement cylinders $(12 \mathrm{~mm} \times 6 \mathrm{~mm})$ were prepared using a steel mold. The surfaces of cement cylinders were smoothed using sandpaper prior to testing. Specimen were loaded into a universal servo-hydraulic test machine (Model 8521, Instron, Norwood, MA) and tested in axial compression at a speed of $0.1 \mathrm{~mm} \mathrm{~s}^{-1}$ until failure. The maximum compressive stress and Young's elastic modulus were calculated. Each cement group was measured in triplicate.

\section{Release of MTX and degradation of cement discs}

Following curing, cement discs $(5 \mathrm{~mm} \times 6 \mathrm{~mm})$ were submerged into $1.5 \mathrm{~mL}$ of deionized distilled water in sealed tubes and maintained at $37{ }^{\circ} \mathrm{C}$. At predetermined time points, eluents were collected and replaced with the same amount of deionized distilled water. Eluents collected were frozen at $-80{ }^{\circ} \mathrm{C}$ till use. At the completion of the release study, cement disc residues were dried, and total mass loss calculated. The disc weight before soaking was measured as $W_{1}$, the disc weight after soaking was measured as $W_{2}$, the weight loss: $\Delta W=\left(W_{1}-W_{2}\right) / W_{2}$. The released MTX was measured by MTX ELISA kit (Endo Life) following manufacturer's instruction. The total percentage released was calculated based on the known initial MTX content. Each group consisted of 3 discs.

\section{Cell viability}

MCF7 cell line was derived from a patient with breast cancer. MCF7 cell line has bone metastasis capability and is sensitive to MTX treatment. MC3T3 is a murine pre-osteoblastic cell line that has been used for cell biocompatibility evaluation. MCF 7 cells were cultured in DMEM medium, supplemented with $5 \%$ fetal bovine serum (FBS, Invitrogen), insulin and penicillin and streptomycin (Life Technologies, Gaithersburg, MD, USA). MC3T3 
cells were cultured in $\alpha$-MEM medium supplemented with $10 \%$ FBS, $10 \mathrm{mM} \beta$-Glycerophosphate (Sigma Aldrich), and antibiotics. Cells were seeded into 48-well plates at concentration of $1.0 \times$ $10^{5}$ per well. After cell attachment, eluents collected at different time points were passed through a syringe filter for sterilization, and $30 \mu \mathrm{l}$ of the eluent was added into $270 \mu \mathrm{l}$ culture medium (final $10 \%, \mathrm{v} / \mathrm{v}$ ) and continue cell culture for three days. Cells cultured in the absence of eluent were included as controls. At the end of the culture, cell viability was evaluated by MTT (3-(4,5-Dimethylthiazol-2-yl)- 2,5-diphenyltetrazolium bromide) activity in cell lysates.

\section{Statistical analysis}

All values are expressed as mean \pm standard deviation. Data were analyzed by One-way Analysis of Variance (ANOVA) with post-hoc Tukey-Kramer test. Statistical significance was set to be $p<0.05$.

\section{Results}

\section{Setting time, injectability and cement degradation}

As shown in Table 1, the setting time was significantly extended in $0.5 \%$ MTX-P (45.7 $\pm 1.9 \mathrm{~min})$ and $1 \%$ MTX-P (49.5 $\pm 3.4 \mathrm{~min})$ as compared to control-P (11.8 $\pm 1.2 \mathrm{~min})$, respectively $(p<0.05)$. Through MTX-P was injectable, but the injectability was reduced as compared to P-DCPD $(p<0.05)$. In addition, the loading of MTX at current concentration $(0.5 \%$ and $1 \%$, respectively) has little impacts on cement degradation rate (\% of weight loss) 28 days after MTX elution study.

\section{SEM analysis}

SEM was used to analyze the surface morphology of MTX-P scaffolds (Fig. 1D-F). As compared to control-P, MTX loading increased the surface roughness and microcracks.

\section{MicroCT analysis}

The representative images of 3D reconstructed P-DCPD scaffolds were shown in Fig. 6. Scaffolds were fabricated with an interconnective porous structure, with a pore size distribution ranging from $<10-120 \mu \mathrm{m}$. MTX-loading changed both the PDCPD scaffold surface morphology (Fig. 1) and the inner pore characteristics. As shown in Table 2, the average pore sizes of $0.5 \%$ MTX-P (70.8 \pm $38.3 \mu \mathrm{m})$ and $1 \%$ MTX-P $(71.8 \pm 21.1 \mu \mathrm{m})$ were slightly reduced, as compared with that of control-P $(110 \pm 60 \mu \mathrm{m})$. MTX loading also changed the pattern of pore size distribution (Fig. 2). There was a significantly lower percentage of pore size in the range of $>90 \mu \mathrm{m}$ for the MTX-P scaffold (29.84\% and 29.14\% for 0.5\% MTX-P and 1\% MTX-P, respectively) than control-P (59.43\%, $p<0.05)$.

\section{Mechanical strength}

A significant reduction of the mechanical strength (16.49 \pm $2.43 \mathrm{MPa}$ ) and Young's modulus (1700.75 $\pm 351.22 \mathrm{MPa})$ was observed in $0.5 \%$ MTX-P, as compared to control-P (53.09 \pm 8.64 MPa and $3170.72 \pm 165.37 \mathrm{MPa}$, respectively, $p<0.05)$, that was more apparent in $1 \%$ MTX-P $(8.68 \pm 6.98 \mathrm{MPa}$ and $918.63 \pm 609.34 \mathrm{MPa}$, respectively, Fig. 3).

Table 1 Setting time, injectability and degradation rate (\%)

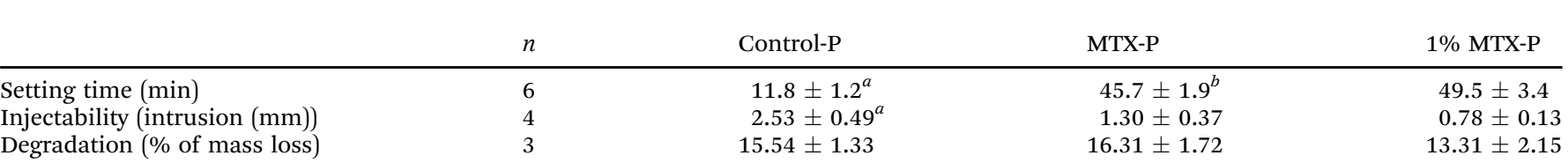

${ }^{a} p<0.05$ between Control-P and MTX-P. ${ }^{b} 0.5 \%$ MTX-P and 1\% MTX-P.

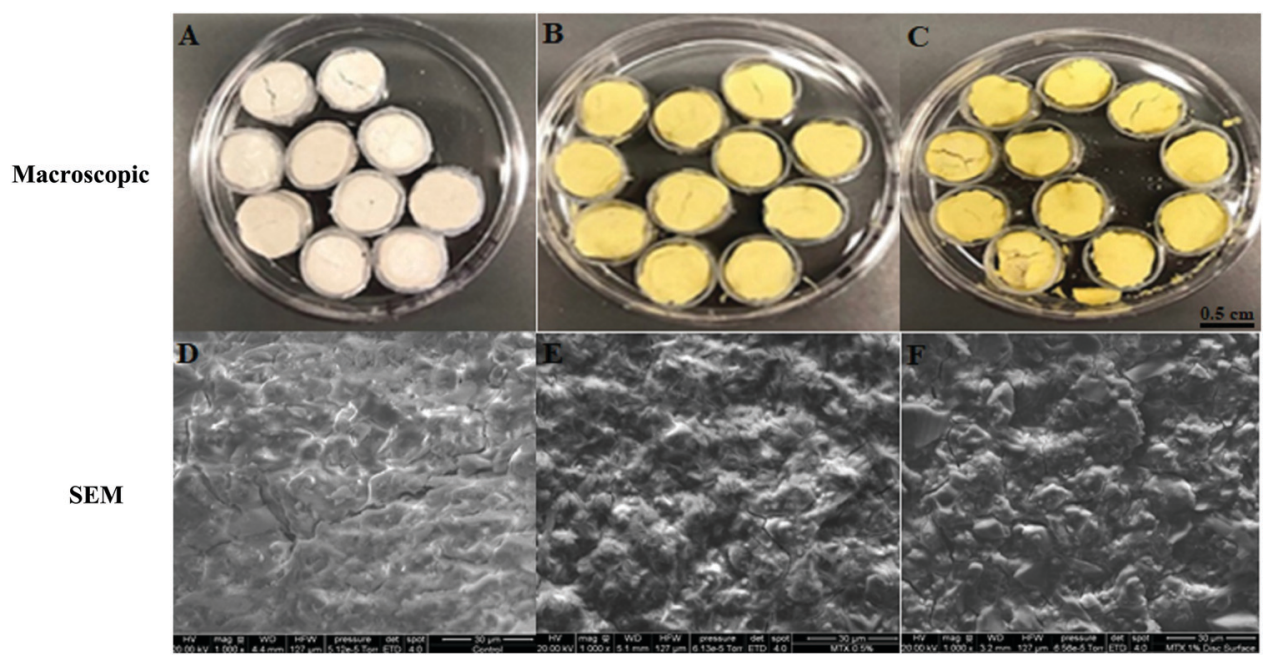

Fig. 1 Microscopic and SEM $(\times 1000)$ appearance of MTX-P scaffolds (A and D) Control-P; $(B$ and E) $0.5 \%$ MTX-P, and (C and F) 1\% MTX-P. 
Table 2 MicroCT-based quantitative MTX-P scaffold morphology analysis $(n=4)$

\begin{tabular}{lllrl}
\hline Sample & Pore size $(\mu \mathrm{m})$ & Porosity $(\%)$ & Tb.Th $(\mathrm{mm})$ & Tb.Sp $(\mathrm{mm})$ \\
\hline Control-P & $110 \pm 60$ & $1.62 \pm 2.9$ & $0.3 \pm 0.27$ & $0.06 \pm 0.12$ \\
$0.5 \%$ MTX-P & $70.8 \pm 38.3$ & $2.71 \pm 1.71$ & $0.27 \pm 0.28$ & $0.08 \pm 0.07$ \\
$1 \%$ MTX-P & $71.8 \pm 21.1$ & $2.66 \pm 3.0$ & $0.27 \pm 0.02$ & $0.07 \pm 0.03$
\end{tabular}

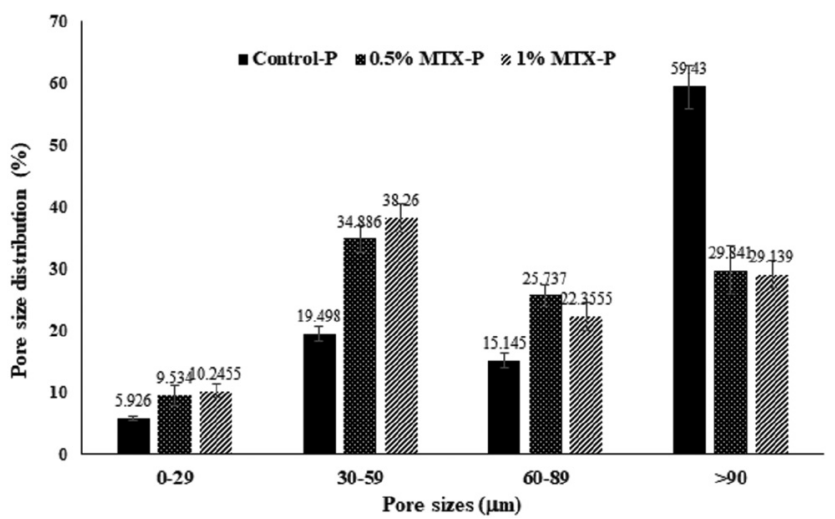

Fig. 2 Pore size distribution of MTX-P scaffold from MicroCT analysis. The 3-D images of scaffolds were formed by overlapping 54 slices of scanned images.

\section{MTX release}

The release of MTX from P-DCPD up to 28 days was studied and the results shown in Fig. 4. A very low percentage (\%) of MTX loaded was released from $0.5 \%$ MTX-P $(1.03 \pm 0.06 \%)$ that was higher than that from $1 \%$ MTX-P $(0.52 \pm 0.03 \%, p<0.05)$. There was a statistically significant difference of the between the $\%$ of release of MTX between 0.5\%MTX-P and 1\%MTX-P at all given time points (Fig. 4A, $p<0.05$ ). The absolute concentration of MTX released from $0.5 \%$ MTX-P was higher at the time of $24 \mathrm{~h}\left(3877 \pm 170 \mathrm{ng} \mathrm{m}{ }^{-1}\right)$ than that of $1 \%$ MTX-P $(2394 \pm$ $\left.120 \mathrm{ng} \mathrm{ml}^{-1}, p<0.05\right)$. Starting from $48 \mathrm{~h}$, the concentration of MTX released from 1\% MTX-P was gradually increased, and a much higher MTX release was observed in 1\%MTX- P in day 21 $\left(553.38 \pm 147.03 \mathrm{ng} \mathrm{ml}{ }^{-1}\right)$ and day $28\left(526.21 \pm 81.93 \mathrm{ng} \mathrm{ml}^{-1}\right)$ as compared to $0.5 \%$ MTX-P $\left(21.44 \pm 3.48 \mathrm{ng} \mathrm{ml}^{-1}\right.$ and $17.24 \pm$ $4.02 \mathrm{ng} \mathrm{ml} \mathrm{m}^{-1}$, respectively, Fig. $4 \mathrm{~B}, p<0.05$ ).

\section{Cell viability}

Eluents from control-P had no negative impacts on cell viability of both MCF 7 and MC3T3 cells. As shown in Fig. 5, eluents from both $0.5 \%$ MTX-P and 1\% MTX-P significantly reduced cell viability of MCF 7 cells as compared to control-P $(p<0.05)$. However, the significant reduction of cell viability at day 21 and day

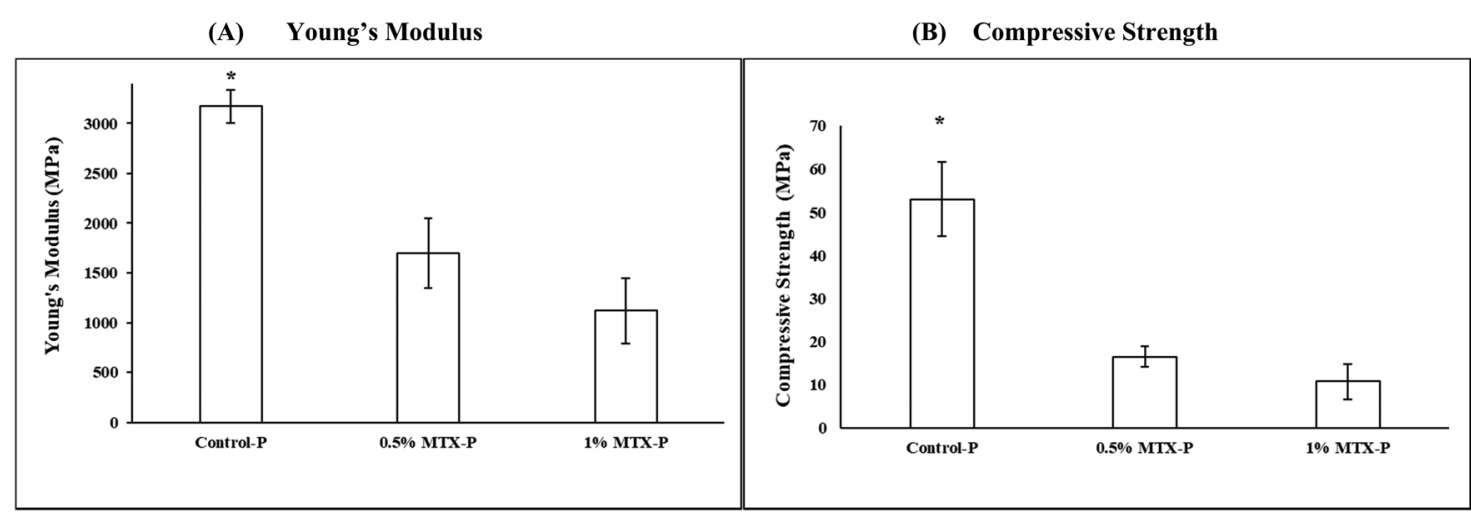

Fig. 3 Mechanical strength measurement of (A) Young's Modulus and (B) Compressive strength. Statistical differences between drug free control (P) and both $0.5 \%$ MTX-P and $1 \%$ MTX-P were indicated by $\left.{ }^{*}\right)(n=3, p<0.05)$. No statistical difference between $0.5 \%$ MTX-P and $1 \%$ MTX-P was found.
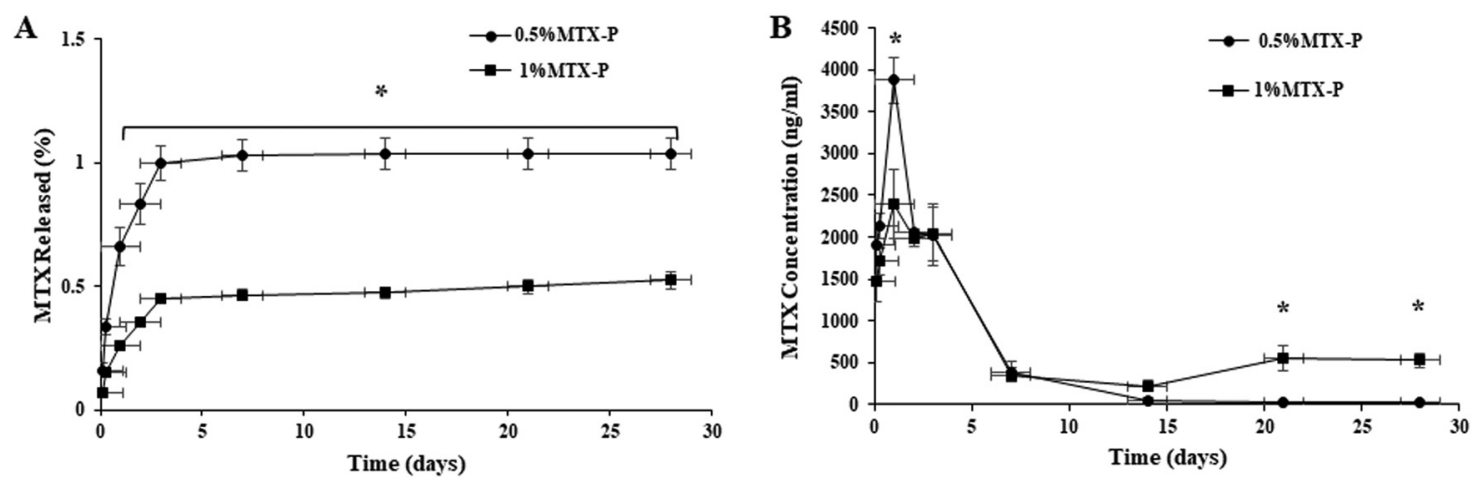

Fig. 4 MTX release profiles (A) cumulative release (\% of initial load) of MTX from MTX-P cement matrices over the course of 28 days. A statistical difference was found between 0.5\%MTX-P and 1\% MTX-P cement at all given time points, and (B) cumulative release (absolute concentration of MTX released) from MTX-P cement matrices over the course of 28 days. Statistical differences were found between $0.5 \%$ MTX-P and $1 \%$ MTX-P cement at 1 day, 21 and 28 days, respectively $(n=3, * p<0.05)$. 


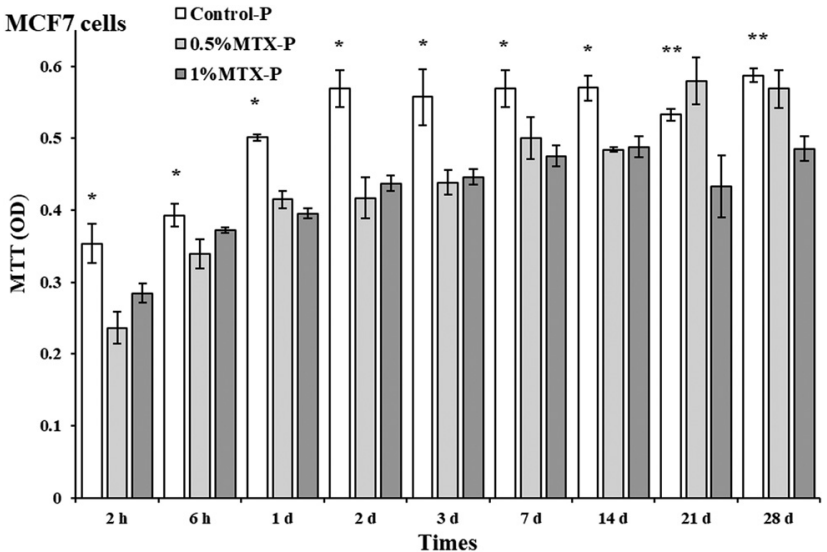

Fig. 5 Cell viability (MTT) of MCF 7 cells and MC3T3 cells when exposed to $10 \%$ eluents collected from MTX-loaded P-DCPD cements at given times; control: eluents from MTX-free P-DCPD cements. Cells were cultured three days before tests. ( $n=3,{ }^{*} p<0.05$ between control and MTX-P, ** between $0.5 \%$ MTX and $1 \%$ MTX).
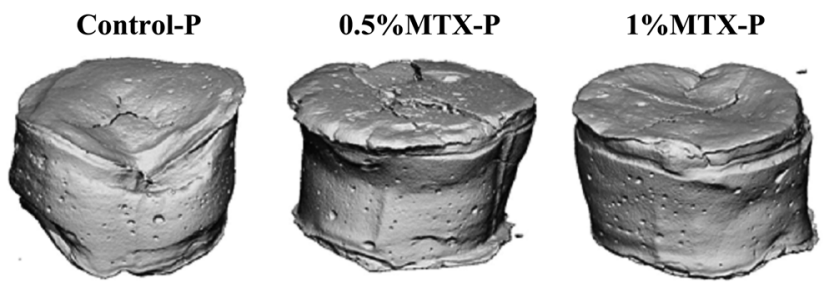

Fig. 6 Representative 3D rendering images of the Control-P, 0.5\%MTX-P and 1\%MTX-P scaffolds determined by micro-CT (voltage: $70 \mathrm{kVp}$; current: $114 \mu \mathrm{A}$ ).

28 was only observed in eluents from 1\% MTX-P because of much higher amount of MTX released (Fig. 4). Interestingly, MT3T3 cells were less sensitive to MTX-containing eluents. Eluents from MTX-P (both $0.5 \%$ and $1 \%$ ) had no effects on cell viability of MC3T3 cells except on the time of day 1 and 2 . The reduction of cell viability observed in these two time points might be due to the higher MTX concentration in the eluents $\left(>2 \mu \mathrm{g} \mathrm{ml}{ }^{-1}\right.$, Fig. 4).

\section{Discussion}

A new injectable P-DCPD was developed in our lab that is mechanically strong and with excellent anti-washout capability. ${ }^{24}$ The key molecular structural difference between P-DCPD and classical DCPD is that P-DCPD is composed of interconnected P-DCPD crystals by interlocking to the polyphosphate chains, while DCPD is composed of a package of DCPD crystal particles with weak mutual bonding. ${ }^{24}$

DCPD has been proposed as an alternative to PMMA for local drug delivery. ${ }^{18}$ Many studies have been aimed at improving DCPD properties capable of delivering drugs in a sustained pattern but with limited success. ${ }^{37}$ Yang et $a .^{38}$ reported an initial burst release of MTX from CPCs doped with 0-1\% MTX, and the release rate was faster in vivo than in vitro. An understanding of the elution profile of MTX from P-DCPD is essential for the prediction of its future in vivo performance. In this study, we showed a unique slow and sustained release of MTX from P-DCPD (Fig. 4), which is rarely seen in ceramic drug delivery system. ${ }^{18,39}$ The intermolecular interaction of entangled polyphosphate chains of CPP gel with loaded MTX plays a critical role in the MTX release profile observed. ${ }^{40}$ We propose that a slow and sustained MTX release observed might be due to an exclusive distribution of MTX within the P-DCPD matrix. ${ }^{41}$ Instead of reacting with CPP polyphosphate chains, MTX, with a small size $\left(\mathrm{C}_{20} \mathrm{H}_{22} \mathrm{~N}_{8} \mathrm{O}_{5} \cdot \mathrm{H}_{2} \mathrm{O}\right.$, MW: 454.5), is absorbed with calcium and phosphate ions dissolved and released from TTCP and then easily penetrated and integrated into formed crystalline lattice of P-DCPD crystals during setting. ${ }^{41}$ In addition, MTX is soluble only in alkaline solution and insoluble in weak acidic (pH 6.5-7.0 during setting) P-DCPD matrix. Therefore, a slow and sustained MTX release was mainly driven by the slower cement degradation accompanied by the opening of the pores within cement matrix. MTX embedded deep in cement matrix become more available for elution after dissolution of the P-DCPD in the presence of water penetration and when the pores or channels are formed during cement degradation. A sustained release of MTX for months at effective concentration right after MTX-P implantation is clinically desirable and is expected to provide a constant inhibition of tumor growth during bone remolding and repair. As a bone void filler, MTX-P is biodegradable and provides a dynamic interaction between degrading cement with surrounding new bone formation, that was not seen in PMMA cement. ${ }^{9}$ The potential therapeutic efficacy of MTX-P will be further tested in animal tumor model before clinical application.

We also noticed that the net MTX release was much higher from $0.5 \%$ MTX-P than that of $1 \%$ MTX-P, especially at the time of $24 \mathrm{~h}$, followed by a gradually increased MTX release from 1\% MTX-P that is more substantial after 14 days (Fig. 4). In vitro antibiotic release profiles can be affected by many factors including the dose of drug loaded. ${ }^{42}$ We proposed that the observed earlier "higher" release from $0.5 \%$ MTX-P might be due to the differences of surface roughness (Fig. 1), particle size distribution (Fig. 2) and degradation rate (Table 1) caused by different amount of MTX added.

Our data showed that eluents from control-P had no negative impacts on cell viability of both MCF 7 and MC3T3 cells. Eluents collected from both 0.5\% MTX-P and 1\% MTX-P were cytotoxic on MCF 7 cells (Fig. 5). However, only eluents from 1\% MTX-P remained the cytotoxicity to MCF 7 cells after 14 days, indicating that the cytotoxicity observed was dose-dependent that was supported by the data of MTX release profile (Fig. 4). MT3T3 cells were less sensitive to MTX-containing eluents. The cell viability of MC3T3 cells were not affected by eluents from MTX-P unless in the eluents collected at day 1 and 2 (with higher MTX concentration $>2 \mu \mathrm{g} \mathrm{ml} \mathrm{m}^{-1}$ ). Decker et al. had a similar finding for the MTX released from PMMA cement. ${ }^{43}$ The different cytotoxic sensitivity between two cell lines might be due to their difference of proliferation rate since MTX acts as an inhibitor of DNA synthesis. ${ }^{34}$ We propose that MTX-P is clinically desirable for the eradication of residual cancer cells at the margin of surgery resection on the very beginning, while has much less impacts on new bone formation. The potential therapeutic efficacy of MTX-P will be further tested in animal tumor model. 
The unique distribution of MTX had inferior impacts on the setting time and mechanical strength of P-DCPD (Table 1). The setting time was significantly delayed in both $0.5 \%$ MTX-P $(45.7 \pm 1.9 \mathrm{~min})$ and $1 \% \operatorname{MTX}-\mathrm{P}(49.5 \pm 3.4 \mathrm{~min})$. The mechanism might be due to that MTX reduced the diffusion efficiency of calcium and orthophosphates dissolved from TTCP, the first step for setting. ${ }^{24}$ It also can be caused by the integration of MTX within the P-DCPD crystals and/or the inhibition of P-DCPD crystal growth and morphological change of the formed crystals. ${ }^{24,44}$ It is critical to prepare a cement with a suitable setting time (around 10-20 minutes) so that it can set slowly enough for the surgeon to perform implantation but fast enough to prevent delaying the operation time. The cement should be moldable and injectable that enables minimally invasive application. ${ }^{45}$ Therefore, more efforts are needed to tailor many processing parameters such as cement composition, additives, porogens, particle size, drug loading amount, adjustment of gel/powder ratio, among others. ${ }^{15}$

Few studies have been conducted on the impacts of loading MTX on the mechanical strength of CPCs. Usually the mechanical strength of DCPD cements tends to decrease when increasing drug loading does. ${ }^{46-48}$ Yang et $a .^{38}$ reported that the compressive strength of CPC was significantly decreased when 1\% MTX was added, but Liao et al. ${ }^{36}$ described that adding $0.1 \%$ or $0.2 \%$ MTX to CPC had no impacts on the mechanical strength. In current study, a significant reduction of the mechanical strength $(16.49 \pm 2.43 \mathrm{MPa})$ was observed in $0.5 \% \mathrm{MTX}-\mathrm{P}$, as compared to control-P (53.09 $\pm 8.64 \mathrm{MPa}, p<0.05)$, that was more obvious when the concentration of MTX was increased in 1\% MTX-P $(8.68 \pm 6.98 \mathrm{MPa})$. We propose that the inferior mechanical strength observed in MTX-P was mainly caused by the insufficient crystal formation and uneven distribution of solid MTX within P-DCPD matrix. ${ }^{24}$ The inferior impacts of MTX loading on the mechanical strength of P-DCPD remains a key obstacle for the clinical application of load-bearing bone defects. Our current studies demonstrated that the property of the loaded drugs (molecular weight, functional groups and water solubility, etc.) plays a critical role in the mechanical performance of P-DCPD. For example, loading of erythromycin ${ }^{28}$ and/or tobramycin ${ }^{27}$ at high concentration $(10 \% \mathrm{wt} / \mathrm{wt})$ had little impacts on the mechanical performance of P-DCPD. Doxorubicin is one of the effective and broad spectrum anticancer drugs. Data from our current study showed that the handling property and mechanical performance of P-DCPD were negligibly changed by adding doxorubicin ( $5 \% \mathrm{wt} / \mathrm{wt}$, unpublished data). Taken together, we propose that the performance improvements of P-DCPD should be achievable by screening of drug candidates with similar anticancer pharmacology besides the modification of cement processing parameters. ${ }^{15}$

As shown in Table 1, the in vitro degradation rate of control-P $(15.54 \pm 1.33 \%)$ was only marginally changed as compared to either $0.5 \%$ MTX-P $(16.31 \pm 1.72 \%)$ or $1 \%$ MTX-P $(13.31 \pm$ $2.15 \%$ ). DCPD is biodegradable and known to possess the highest solubility among all CPCs at physiological conditions. ${ }^{49}$ Many studies have demonstrated that the degradation rate of DCPD was within a period of $8-52$ weeks both in vitro and in vivo based on experiment settings. ${ }^{50-53} \mathrm{We}^{49}$ currently demonstrated that
P-DCPD has a more stable chemical structure than DCPD as evidenced by much less transformation to HA during setting and evolution. Importantly, P-DCPD has lower zeta potential and less hydrophilicity than that of DCPD because of its entangled and interconnected polyphosphate chains. It is expected that superhydrophilic DCPD undergoes faster dissolution than P-DCPD in an aqueous environment.

There are some limitations to this study. First, MTX-loading significantly reduced the mechanical strength of PDCPD and thus limited its potential application for the load-bearing bone defect filling. Second, MTX loading reduced the pore size. More than $70 \%$ of MTX-P was composed of pore size $<90 \mu \mathrm{M}$, as defined by MicroCT measurement (Fig. 2 and Table 2). The potential impact(s) of MTX loading on cellular behaviors and bone ingrowths warrants further investigation. In addition, more extensive studies are needed to optimize MTX loading, setting time optimization, and drug release adjustment.

\section{Conclusions}

A slow and sustained MTX release from MTX-P was observed. MTX-P is biocompatible and biodegradable. Incorporation of MTX at current amount had inferior impacts on the setting time and mechanical strength of P-DCPD. We propose that the detrimental performance of P-DCPD observed in MTX-P was mainly caused by the insufficient crystal formation and uneven distribution of MTX within P-DCPD matrix. Our data may reveal the future application of MTX-P in the treatment of bone defects after tumor excision. Further material optimization and in vivo validation are required to achieve a clinically applicable product.

\section{Author contributions}

Rahul Vaidya: Conceptualization, methodology; Emily J Ren: Investigation, formal analysis; Tong Shi: Investigation; Angelica Gardia: Investigation, writing-original draft; Weiping Ren: Supervision, validation, writing-review \& editing.

\section{Conflicts of interest}

There are no conflicts to declare.

\section{Acknowledgements}

We acknowledge Mrs Paula Dietz and Dr Yawen Li for the assistance of MicroCT and SEM data analysis.

\section{References}

1 A. S. Gdowski, A. Ranjan and J. K. Vishwanatha, J. Exp. Clin. Cancer Res., 2017, 36, 108.

2 E. Verron, H. Schmid-Antomarchi, H. Pascal-Mousselard, A. Schmid-Alliana and J.-C. Scimeca, Drug Discovery Today, 2014, 19, 1419-1426. 
3 A. Schmid-Alliana, H. Schmid-Antomarchi, R. Al-Sahlanee, P. Lagadec, J.-C. Scimeca and E. Verron, Int. J. Mol. Sci., 2018, 19, 148.

4 M. Levy, T. Smith, A. Alvarez-Perez, A. Back and J. N. Baker, J. Natl. Compr. Cancer Network, 2016, 14, 82-113.

5 A. Myoui and H. Yoshikawa, Clinical calcium, 2008, 18, 1767-1773.

6 I. Pountos and P. V. Giannoudis, Expert Rev. Med. Devices, 2018, 15, 301-311.

7 H. Futani, S. Kamae, K. Atsui, K. Yoh, H. Tateishi and S. Maruo, J. Orthop. Sci., 2002, 7, 262-266.

8 H. Futani, S. Kamae, K. Atsui, K. Yoh and H. Tateishi, J. Orthop. Sci., 2002, 7, 262-266.

9 G. Maccauro, A. Cittadini, M. Casarci, F. Muratori, D. De Angelis, C. Piconi, M. A. Rosa, A. Spadoni, M. Braden and A. Sgambato, J. Mater. Sci.: Mater. Med., 2007, 18, 839-844.

10 G. W. Fröschle, J. Mahlitz, H. U. Langendorff, E. Achilles and J. Pollock, Anticancer Res., 1997, 17, 995-1002.

11 H. Katagiri, K. Sato, M. Takahashi, H. Sugiura, S. Yamamura and H. Iwata, Archives of Orthopaedic and Trauma Surgery, 1997, 116, 329-333.

12 R. Król and S. Radomski, Ortopedia, traumatologia, rehabilitacja, 2003, 5, 319-326.

13 S. Wasserlauf, A. Warshawsky, R. Arad-Yelin, Y. Mazur and R. Salama, Bull. Hosp. Jt. Dis., 1993, 53, 68-74.

14 W. Song, J. Seta, M. K. Eichler, J. J. Arts and B. M. Boszczyk, J. Biomed. Mater. Res., Part B, 2018, 106, 2693-2699.

15 J. Zhang, W. Liu, V. Schnitzler, F. Tancret and J. M. Bouler, Acta Biomater., 2014, 10, 1035-1049.

16 Y. Tanzawa, H. Tsuchiya, T. Shirai, H. Nishida and K. Hayashi, J. Orthop. Sci., 2011, 16, 77-84.

17 M. Gallo, S. Tadier, S. Meille, L. Gremillard and J. Chevalier, Acta Biomater., 2017, 53, 515-525.

18 D. Arcos and M. A. Vallet-Regí, Acta Mater., 2013, 61, 890-911.

19 E. Van Lieshout, G. Van Kralingen, Y. El-Massoudi, H. Weinans and P. Patka, BMC Musculoskeletal Disord., 2011, 12, 34.

20 P. J. Jiang, S. Patel, U. Gbureck, R. Caley and L. M. Grover, J. Biomed. Mater. Res., Part B, 2010, 93, 51-58.

21 X. Wang, H. C. Schroeder and W. E. G. Mueller, Biotechnol. J., 2016, 11, 11-30.

22 R. M. Pilliar, M. J. Filiaggi, J. D. Wells, M. D. Grynpas and R. A. Kandel, Biomaterials, 2001, 22, 963-972.

23 W. Song, J. Seta, R. Kast, G. Auner, L. Chen, D. Markel and W. Ren, J. Am. Ceram. Soc, 2015, 98, 3758-3769.

24 W. Ren, W. Song, S. Yurgelevic and D. C. Markel, J. Mech. Behav. Biomed. Mater., 2018, 79, 226-234.

25 W. E. Brown and L. C. Chow, Int. Am. Assoc. Dent. Res, 1983, 62, 672.

26 A. Criado, S. Yokhana, T. Rahman, S. McCarty, C. Andrecovich, W. Ren and W. K. Yassir, Spine Deformity, 2020, 8, 165-170.

27 E. J. Ren, A. Guardia, T. Shi, P. Begeman, W. Ren and R. Vaidya, Biomed. Mater., 2021, 16, 025019.

28 A. Guardia, T. Shi, T. Bou-Akl, P. Dietz, B. Wu, W. Ren and D. Markel, J. Orthop. Res., 2021, 1-9.
29 Z. Zhou, J. Seta, D. C. Markel, W. Song, S. M. Yurgelevic, X. W. Yu and W. Ren, J. Biomed. Mater. Res., Part B, 2018, 106, 2827-2840.

30 U. Tariq, R. Hussain, K. Tufail, Z. Haider and R. Tariq, Mater. Sci. Eng., C, 2019, 103, 109863.

31 S. S. Abolmaali, A. M. Tamaddon and R. Dinarvand, Cancer Chemother. Pharmacol., 2013, 71, 1115-1130.

32 N. Limelette, M. Ferry, S. Branger, A. Thuillier and C. Fernandez, Ther. Drug Monit., 2003, 25, 81-87.

33 J.-X. Zheng, H. Jiang, L.-P. Xiao, S.-S. Wang and X.-D. Li, Int. J. Clin. Exp. Pathol., 2017, 10, 368-376.

34 E. Prochazka, T. Soukup, M. Hroch, L. Fuksa, E. Brcakova, J. Cermanova, G. Kolouchova, K. Urban, J. Mokry and S. Micuda, International Orthopaedics, 2010, 34, 137-142.

35 K. Anagnostakos, O. Furst and J. Kelm, Acta Orthop., 2006, 77, 628-637.

36 G. Liao, D. Sun, J. Han and J. Tan, Orthopedics, 2014, 37, e906-e910.

37 M. P. Hofmann, A. R. Mohammed, Y. Perrie, U. Gbureck and J. E. Barralet, Acta Biomater., 2009, 5, 43-49.

38 Z. Yang, J. Han, J. Li, X. Li and Z. Li, Orthopedics, 2009, 32, 27.

39 S. M. H. Dabiri, A. Lagazzo, B. Aliakbarian, M. Mehrjoo, E. Finocchio and L. Pastorino, J. Biomed. Mater. Res., Part A, 2019, 107, 2063-2075.

40 Y. Chehreghanianzabi, R. Barua, T. Shi, S. Yurgelevic, G. Auner, D. C. Markel and W. Ren, J. Biomed. Mater. Res., Part B, 2020, 108, 475-483.

41 M. P. Ginebra, C. Canal, M. Espanol, D. Pastorino and E. B. Montufar, Adv. Drug Delivery Rev., 2012, 64, 1090-1110.

42 H. van de Belt, D. Neut, D. R. A. Uges, W. Schenk and H. van, Biomaterials, 2000, 21, 1981-1987.

43 S. Decker, W. Winkelmann, B. Nies and F. van Valen, J. Bone Jt. Surg., Br. Vol., 1999, 81-B, 545-551.

44 M. P. Ginebra, T. Traykova and J. A. Planell, J. Controlled Release, 2006, 113, 102-110.

45 K. L. Low, S. H. Tan, S. H. S. Zein, J. A. Roether, V. Mourio and A. R. Boccaccini, J. Biomed. Mater. Res., Part B, 2010, 94, 273-286.

46 M. H. Alkhraisat, C. Rueda, J. Cabrejos-Azama, J. LucasAparicio, F. T. Marino, J. Torres Garcia-Denche, L. B. Jerez, U. Gbureck and E. L. Cabarcos, Acta Biomater., 2010, 6, 1522-1528.

47 M. Takechi, Y. Miyamoto, K. Ishikawa, M. Nagayama and M. Kon, J. Biomed. Mater. Res., 1998, 39, 308-316.

48 Y. Huang, C. S. Liu, H. F. Shao and Z. J. Liu, Key Eng. Mater., 2000, 192-195, 853-860.

49 R. Barua, C. S. Daly-Seiler, Y. Chenreghanianzabi, D. Markel, Y. Li, M. Zhou and W. Ren, J. Biomed. Mater. Res., Part B, 2021, 1-12.

50 J. M. Kuemmerle, A. Oberle, C. Oechslin, M. Bohner and C. Frei, J. Cranio-Maxillofacial Surg., 2005, 33, 37-44.

51 S. Shadanbaz, J. Walker, T. Woodfield, M. Staiger and G. Dias, J. Mater. Sci.: Mater. Med., 2014, 25, 173-183.

52 F. Tamimi, Z. Sheikh and J. Barralet, Acta Biomater., 2012, 8, 474-487.

53 Z. Xia, L. M. Grover, Y. Huang, L. E. Adamopoulos and U. Gbureck, Biomaterials, 2006, 27, 4557-4565. 